\title{
Testing copula regression against benchmark models for point and interval estimation of tree wood volume in beech stands
}

\author{
Francesco Serinaldi • Salvatore Grimaldi • \\ Mohammad Abdolhosseini · Piermaria Corona • \\ Dora Cimini
}

Received: 31 March 2011/Revised: 18 December 2011/Accepted: 24 January 2012/Published online: 24 February 2012

(C) Springer-Verlag 2012

\begin{abstract}
This study compares copula regression, recently introduced in the forest biometric literature, with four benchmark regression models for computing wood volume $V$ in forest stands given the values of diameter at breast height $D$ and total height $H$, and suggests a set of statistical techniques for the accurate assessment of model performance. Two regression models deduced from the trivariate copulabased distribution of $V, D$, and $H$ are tested against the classical Spurr's model and Schumacher-Hall's model based on allometric and geometric concepts, and two regression
\end{abstract}

Communicated by G. Kändler.

F. Serinaldi $(\bowtie)$

School of Civil Engineering and Geosciences, Newcastle

University, Newcastle Upon Tyne NE1 7RU, UK

e-mail: francesco.serinaldi@ncl.ac.uk

F. Serinaldi

Willis Research Network, 51 Lime St., London EC3M 7DQ, UK

S. Grimaldi · P. Corona · D. Cimini

Dipartimento DIBAF, Università della Tuscia, Via S. Camillo de

Lellis snc, 01100 Viterbo, Italy

S. Grimaldi

Honors Center of Italian Universities, H2CU, Sapienza

Università di Roma, Via Eudossiana 18, 00184 Roma, Italy

S. Grimaldi

Department of Mechanical and Aerospace Engineering,

Polytechnic Institute of New York University,

Six MetroTech Center, Brooklyn, NY 11201, USA

M. Abdolhosseini

Department of Water Engineering, College of Agriculture, Isfahan University of Technology, 84156-83111 Isfahan, Iran models that rely on Box-Cox transformed variables and are in a middle ground, in terms of model complexity, between copula-based regression and classical models. The accuracy of the point estimates of $V$ is assessed by a suitable set of performance criteria and the nonparametric sign test, whereas the associated uncertainty is evaluated by comparing empirical and nominal coverage probabilities of the prediction intervals. Focusing on point estimates, the Schumacher-Hall's model outperforms the other models in terms of several performance criteria. The sign test points out that the differences among the models that involve $D$ and $H$ as separate covariates are not definitely significant, whereas these models outperform the models with a single covariate. As far as the interval estimates are of concern, the four benchmark models provide comparable interval estimates. The copula-based model with parametric marginals is definitely outperformed by its competitors according to all criteria, whereas the copula-based model with nonparametric marginals provides quite accurate point estimates but biased interval estimates of $V$.

Keywords Fagus sylvatica. Weighted regression . Box-Cox transformation - Copula regression . Normal quantile transformation - Uncertainty

\section{Introduction}

The quality of good forest management and planning relies on the accuracy and precision of forest resource assessment. Therefore, particular attention is paid to the construction of prediction relationships for tree volume $V$ or biomass in forest stands at national and local level (Tabacchi et al. 2011). According to West (2009), this task can be accomplished through three types of techniques: 